\title{
From Revolution to Evolution: The Glutamate Hypothesis of Schizophrenia and its Implication for Treatment
}

\author{
Bita Moghaddam ${ }^{\star 1}$ and Daniel Javitt ${ }^{2}$ \\ ${ }^{1}$ Department of Neuroscience and Psychiatry, University of Pittsburgh, Pittsburgh, PA, USA; ${ }^{2}$ Department of Psychiatry and \\ Neuroscience, Nathan Kline Institute for Psychiatric Research/New York University School of Medicine, Orangeburg, NY, USA
}

Glutamate is the primary excitatory neurotransmitter in mammalian brain. Disturbances in glutamate-mediated neurotransmission have been increasingly documented in a range of neuropsychiatric disorders including schizophrenia, substance abuse, mood disorders, Alzheimer's disease, and autism-spectrum disorders. Glutamatergic theories of schizophrenia are based on the ability of N-methyl-D-aspartate receptor (NMDAR) antagonists to induce schizophrenia-like symptoms, as well as emergent literature documenting disturbances of NMDAR-related gene expression and metabolic pathways in schizophrenia. Research over the past two decades has highlighted promising new targets for drug development based on potential pre- and postsynaptic, and glial mechanisms leading to NMDAR dysfunction. Reduced NMDAR activity on inhibitory neurons leads to disinhibition of glutamate neurons increasing synaptic activity of glutamate, especially in the prefrontal cortex. Based on this mechanism, normalizing excess glutamate levels by metabotropic glutamate group $2 / 3$ receptor agonists has led to potential identification of the first non-monoaminergic target with comparable efficacy as conventional antipsychotic drugs for treating positive and negative symptoms of schizophrenia. In addition, NMDAR has intrinsic modulatory sites that are active targets for drug development, several of which show promise in preclinical/early clinical trials targeting both symptoms and cognition. To date, most studies have been done with orthosteric agonists and/or antagonists at specific sites. However, allosteric modulators, both positive and negative, may offer superior efficacy with less danger of downregulation.

Neuropsychopharmacology Reviews (2012) 37, 4-15; doi: I0.1038/npp.20 I I. I8I; published online 28 September 20II

Keywords: NMDA receptors; antipsychotic drugs; cognition; dopamine

\section{INTRODUCTION}

Fifty years ago, when the ACNP was first convened, it was an exciting time for new treatment development in psychiatry. In schizophrenia, a new era of treatment was ushered by the fortuitous finding that chlorpromazine and other phenothiazines showed dramatic effects in control of psychosis. Implementation of these treatments permitted deinstitutionalization of large numbers of patients with schizophrenia, and allowed a substantial number of chronically disabled patients to resume relatively normal lives. This was followed shortly thereafter with seminal findings linking effective doses of antipsychotic drugs to

${ }^{*}$ Correspondence: Dr B Moghaddam, Department of Neuroscience and Psychiatry, University of Pittsburgh, A210 Langley Hall, Pittsburgh, PA 15260, USA, Tel: +412 624 2653, Fax: +412 624 9198, E-mail: bita@pitt.edu

Received 1 March 2011; revised 21 July 2011; accepted 21 July 2011 blockade of the recently discovered D2-type dopamine receptor (Seeman et al, 1975; Creese et al, 1976). Pharmaceutical companies quickly exploited these findings by developing large families of phenothiazine and nonphenothiazine antipsychotics, all showing similar efficacy and with side effect profiles modulated by binding profile across a wide variety of receptor types.

Fifty years later, pharmacological treatment of schizophrenia remains virtually unchanged (Lieberman et al, 2005). The most efficacious antipsychotic drug is clozapine, developed in 1961. All attempts to develop an equally effective compound free of its hematological and orthostatic side effects so far have failed. The failure is most obvious in the case of negative symptoms and cognitive deficits, which remain as key predictors of functional disability (Anderson et al, 1996; Goldberg and Weinberger, 1996; Green and Nuechterlein, 1999; Goldberg et al, 2003; Kirkpatrick et al, 2006). However, even positive symptoms of schizophrenia 
persist despite an aggressive antipsychotic treatment in a significant number of individuals (Foussias and Remington, 2010). Perhaps most disappointingly, not only have compounds directed at $\mathrm{D} 2$ receptors failed to ameliorate core symptoms of schizophrenia in a great many individuals, but even compounds directed at some of the most obvious closely related sites, such as serotonin 5-HT2A receptors, have no or inferior efficacy compared with typical antipsychotics such as haloperidol (Geyer et al, 1999b; Meltzer et al, 2004; Marder, 1999). A half century after the initial discovery of antipsychotics, the field finds itself in need not only of alternative medications but also alternative targets (Abbott, 2010). Selection of these targets must be guided by sound etiological theories, as well as by practical considerations such as 'drug ability' and stability of effect.

The glutamate synapse has emerged as one of the most prominent targets in this context (Javitt, 2004; Moghaddam, 2004). This is due in part to the fact that biological evidence at several levels supports an involvement for glutamate neurotransmission in the etiology and pathophysiology of the disease. More importantly, the glutamate synapse is a target-rich environment containing a large number of presynaptic, postsynaptic, and regulatory proteins that represent appropriate targets for drug development (Moghaddam, 2003; Marek et al, 2010). Here, we review the progression of scientific discovery and theoretical thinking that has moved the glutamate hypothesis of schizophrenia from a neurotransmitter theory into the practical arena of target identification and animal modeling.

\section{THE REVOLUTION}

The dopamine hypothesis of schizophrenia, which remains the most prominent theory in the field, can be seen as originating from the fortuitous discovery of the antipsychotic effects of chlorpromazine in the mid-1950s. So too, glutamatergic theories can be dated to a precise observation, in this case the synthesis in the late 1950s of the dissociative anesthestics phencyclidine (PCP) and ketamine (Chen and Weston, 1960), followed shortly thereafter by the demonstration of their psychotogenic potential in humans (Luby et al, 1962), the discovery of the PCP receptor (Zukin and Zukin, 1979), and finally the discovery that these compounds function by blocking the $N$-methyl-D-aspartate receptor (NMDAR) channel (Javitt and Zukin, 1991). PCP and ketamine induced negative symptoms and cognitive dysfunction similar to that of schizophrenia, suggesting that this model may be particularly relevant to persistent, pooroutcome forms of schizophrenia.

Historically, attempts have been made to attribute PCP- and ketamine-induced psychosis to a wide variety of targets including dopaminergic, monoaminergic, cholinergic, GABAergic, opiatergic, sigma (Javitt and Zukin, 1991), and, most recently, to high D2 receptors (Seeman, 2010). However, the behavioral effects of NMDAR antagonists that are relevant to schizophrenia persist in the absence of dopamine activity (Carlsson and Carlsson, 1989; Adams and Moghaddam, 1998) or dopamine antagonists (Krystal et al, 1995). Furthermore, both the absolute concentrations and the rank-order potency with which a range of compounds induce psychotomimetic effects in humans and animal models conforms to their rank order of potency at NMDAR but not other receptor types (Javitt and Zukin, 1991; Seeman, 2010).

To date, all compounds that bind to the PCP site of the NMDAR have been found to induce psychosis when given to humans, whereas the same is not true for proposed alternative non-NMDAR-related targets, so that NMDAR blockade by these agents appears both necessary and sufficient to explain their psychotomimetic effects. Moreover, the effects do not appear to be unique to agents that inhibit NMDAR via the PCP site. Thus, antagonists at both the glutamate binding site (eg CGS-19755) and the glycine modulatory site (eg, CP-101,606) also induce psychotomimetic effects when administered clinically (Muir et al, 1995; Preskorn et al, 2008). Finally, it has been observed recently that psychosis related to systemic lupus erythematous and other autoimmune disorders may be due to the production of CNS-penetrant anti-NMDAR antibodies (Omdal et al, 2005), providing unexpected support to NMDAR models of endogenous psychosis.

The NMDAR model may be considered revolutionary, not only because it proposes a different set of targets than would be predicted by more traditional monoaminergic models, but because it proposes a fundamental reconceptualization of what brain regions to target and what assays may be most effective for continued drug development. In particular, dopamine projections in the brain are relatively discrete. In dopaminergic models, therefore, symptoms are seen as arising from dysfunction within a limited number of brain regions, such as dorsolateral prefrontal cortex (Lesh et al, 2011) or striatum (Simpson et al, 2010), with secondary top-down dysregulation elsewhere in the brain.

In contrast to the limited range of dopamine neurotransmission, all cortical efferents and the majority of cortical afferents, and cortico-cortical connections are glutamatergic. In glutamatergic models, therefore, deficits are seen as distributed throughout cortical and subcortical regions, within involvement of sensory as well as higher cortical brain regions (Javitt, 2009b). For example, mismatch negativity (MMN), an event-related potential that indexes brain function at the level of auditory cortex, has been shown repeatedly to be abnormal in schizophrenia (Javitt et al, 1993; Naatanen and Kahkonen, 2009). Deficits similar to those observed in schizophrenia are induced by ketamine administration in normal volunteers (Krystal et al, 1994; Umbricht et al, 2000; Heekeren et al, 2008) and by local administration of NMDAR antagonists into auditory cortex of awake, behaving primates (Javitt et al, 1996). Most recently, similar effects have been reported in rodents (Ehrlichman et al, 2008; Tikhonravov et al, 2008), suggesting that measures such as MMN may be used as translational biomarkers for future drug development research. 
Nevertheless, the observation that symptoms, cognitive deficits, and neurophysiological indices of schizophrenia can be reproduced by blocking NMDAR receptors does not, in itself, explain either how such deficits arise or how they best can be treated. In the simplest versions of the NMDAR models, the primary goal of treatment would be the restoration of function at the NMDAR itself. However, in many, if not most, medical conditions, the target of treatment may not be the site of dysfunction. Thus, a goal of ongoing research has been to delineate, not only potential causes of NMDAR dysfunction, but also the steps leading from NMDAR dysfunction to psychosis and cognitive impairment. This research has led to an evolution in the conceptualization of glutamatergic dysfunction over the past 20 years, and elaboration of targets beyond the NMDAR itself.

\section{THE EVOLUTION}

Despite the conceptual simplicity of the NMDAR model, ie ongoing NMDAR hypofunction leads to expression of schizophrenia symptoms, two broad classes of questions with regard to NMDAR dysfunction remain unanswered. First, what causes NMDAR dysfunction on an etiological level and second, what approaches may be most effective in reversing underlying abnormalities. Research into both causes and treatments continues to evolve. Here, we divide the current ideas and therapeutic approaches into two broad categories of presynaptic and postsynaptic hypotheses.

\section{Presynaptic Hypotheses}

On the presynaptic level, the most obvious potential cause of NMDAR dysfunction would be a reduction in overall glutamatergic tone in the brain, leading to a global deficit in glutamatergic neurotransmission. However, while some findings of reduced CSF glutamate levels were reported (Kim et al, 1980), ultimately these were not confirmed (Javitt and Zukin, 1991), suggesting that more complex disturbances in glutamatergic function might be involved. In fact, over the last 20 years, it has been increasingly demonstrated that hyper, rather than hypo, glutamatergic function, potentially mediated through activation of AMPA receptors may be critical in schizophrenia, and that ideal treatment approaches may reduce rather than increase presynaptic glutamate levels (Moghaddam, 2003).

One key finding leading to the glutamate hyperactivity theory was that, in awake animals (but not in brain slice preparations or anesthetized animals), systemic injection of NMDAR antagonists at doses that impaired cognitive functions and produced motor stereotypy increase glutamate efflux in the prefrontal cortex (Liu and Moghaddam, 1995; Moghaddam et al, 1997; Moghaddam and Adams, 1998; Lorrain et al, 2003). This increase in the extracellular levels of glutamate had functional significance because blockade of AMPA receptors reduced the motoric and cognitive detriments of NMDAR blockade (Moghaddam et al, 1997). Thus, NMDAR antagonists appeared to increase the release of glutamate at some synapses, which then abnormally increased glutamate neurotransmission at nonNMDAR, in particular AMPA receptors (Figure 1). This finding, therefore, suggested that behavioral consequences of NMDAR deficiency is not due to a generalized 'glutamate hypofunction' but dysregulation of glutamate neurotransmission that may potentially involve NMDAR hypofunction but excessive activity of non-NMDA receptors.

Two additional lines of evidence from animal and human studies supported this mechanism. One was that neuronal activity in the PFC was generally enhanced by NMDAR antagonists in human fMRI studies measuring metabolic activation in PFC regions (Breier et al, 1997; Vollenweider et al, 1997) and single unit recordings in awake rodents measuring random spiking of spontaneously active neurons (Jackson et al, 2004) (Figure 1). Although this increased activity may be interpreted counterintuitive to a state of 'hypofrontality' in schizophrenia, the enhanced spike activity led to a disorganized pattern of activity essentially adding 'noise' and interrupting the ability of cortical neurons to process relevant information.

A second line of evidence was that reducing the release of glutamate by metabotropic glutamate group $2 / 3$ receptor agonists also reduced the behavioral and cellular effects of NMDAR antagonists (Moghaddam and Adams, 1998; Krystal et al, 2005). These glutamate receptors are localized extrasynaptically including on presynaptic terminals. Activation of these receptors by exogenous agonists reduces activated release of glutamate (Battaglia et al, 1997; Schoepp et al, 1997). Rodent studies showed that these exogenous agonists reduced NMDAR antagonist-activated release of glutamate and cortical hyperactivity at the same time they ameliorated the aberrant behavioral effects of these antagonists, including PCP and MK801 (Moghaddam and Adams, 1998; Cartmell et al, 1999; Cartmell et al, 2000; Homayoun et al, 2005). Proof of concept studies in healthy volunteers showed that $\mathrm{mGlu} 2 / 3$ receptor agonists also reduced some of the cognitive impairing effects of ketamine in healthy volunteers (Krystal et al, 2005). Collectively, these studies showed that the excitatory consequences of NMDAR hypofunction may mediate some of the behavioral effects of this treatment and, more importantly, reducing the presynaptic output of glutamate receptors by presynaptic autoreceptors such as mGlu2 receptors or by targeting synthetic enzymes such as glutaminase (Gaisler-Salomon et al, 2009) provide novel targets for treatment of symptoms of schizophrenia. Clinical trials based on this concept have so far been encouraging. The first published study demonstrated comparable efficacy between an mGlu2/3 receptor agonist and the atypical antipsychotic drug olanzopine for treating negative and positive symptoms (Patil et al, 2007) without the metabolic and motor-related side effects generally associated with antipsychotic drug. A subsequent trial was inclusive because both the mGlu2/3 agonist and olanzopine did not significantly separate from 


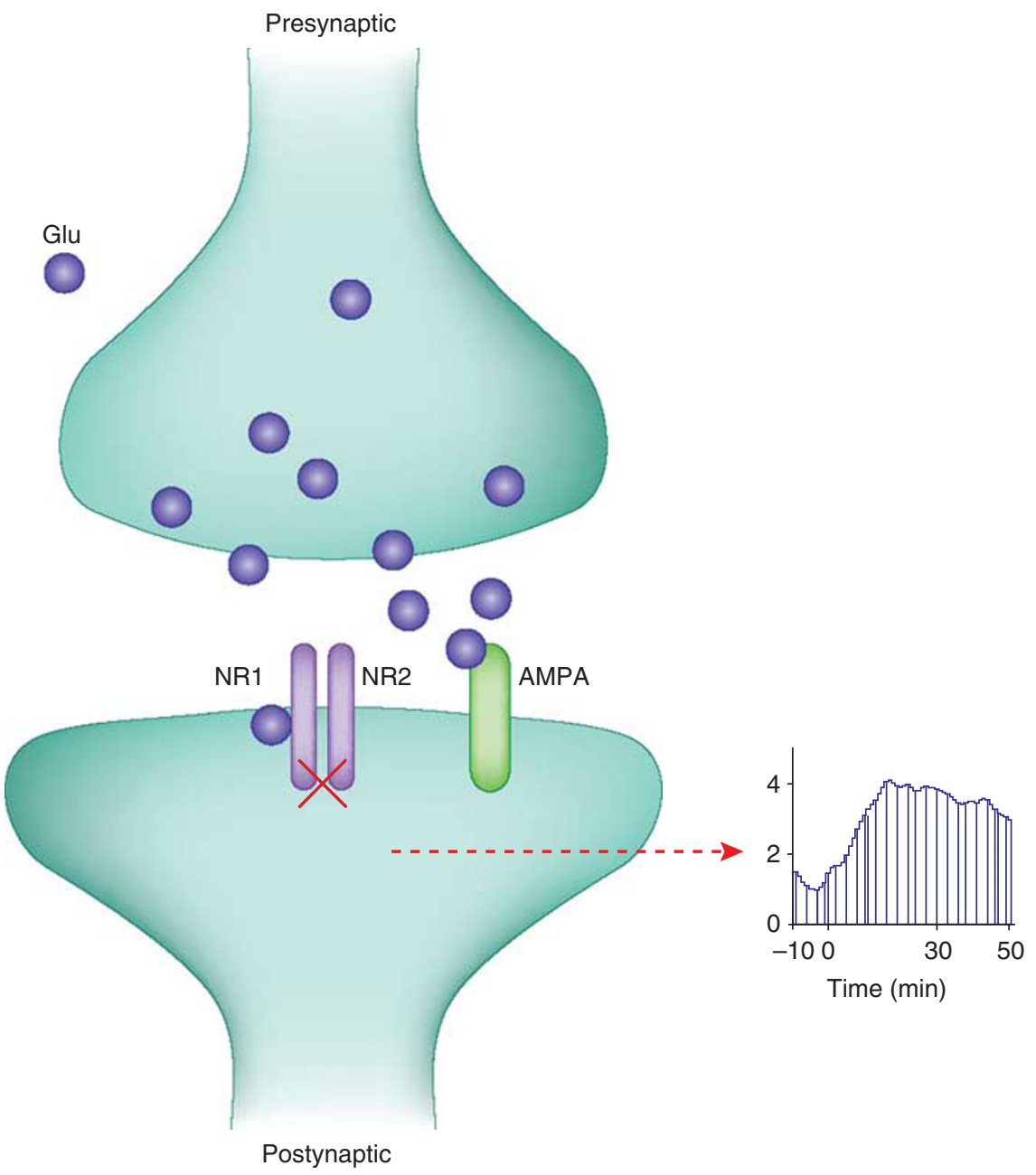

Figure 1. One of the downstream consequences of NMDAR inhibition is increased availability of glutamate (see text and Figure 2 for potential mechansims that can cause this effect). This increase causes excess activity of AMPA receptors and enhanced postsynaptic spiking of cortical principle cells at rest.

placebo (Kinon et al, 2011). A recent long-term (6 months) safety trial comparing mGlu2/3 agonist with atypical antipsychotic standard of care showed that the mGlu2/3 agonist is not associated with increased seizure rates and has an efficacy profile that is consistent with an active antipsychotic compound (B Kinon, personal communication). There were, however, more patients who discontinued mGlu2/3 agonist treatment owing to reduced efficacy. This may be expected given that continued use of orthosteric agonist may lead to receptor desensitization and thus lack of efficacy. Therefore, increasing the dose or shifting to targets that allosterically modulate this receptor may be necessary for chronic use.

The critical question in this context has been what is the mechanism by which an NMDAR antagonist increases and thus disorganizes the firing of cortical neurons? The most straightforward mechanism is the so-called cortical disinhibition process (Homayoun and Moghaddam, 2007b). Figure 2 depicts this model at its simplest form. It is well established that the activity of hippocampal and neocortical principal (pyramidal) neurons is under the control of GABA interneurons. Without this GABAergic inhibition, excitatory inputs onto pyramidal neurons would cause a chain reaction of ever increasing activation. The regulation or stabilization of the firing of pyramidal neurons by GABA interneurons is critical for coordination of cell assemblies that support cortical-mediated behaviors (Buzsaki et al, 2004). One classic example of GABAergic influence is the feed-forward inhibition where the effect of afferent excitation on a pyramidal neuron is dampened by co-activation of GABA interneurons that synapse onto the same pyramidal neuron. Thus, increased discharge of an interneuron results in decreased discharge of pyramidal neurons. Accordingly, processes that inhibit the discharge of GABA neurons excite or 'disinhibit' pyramidal neurons.

In the neocortex and hippocampus, some subtypes of interneurons have a lower threshold for action potential generation compared with pyramidal cells (Csicsvari et al, 1998; Maccaferri and Dingledine, 2002). This more depolarized state of interneurons would dictate that more NMDAR channels, which are voltage gated, are open on these neurons. Given this, when the system is exposed to an 


\section{a Excitatory input}

b

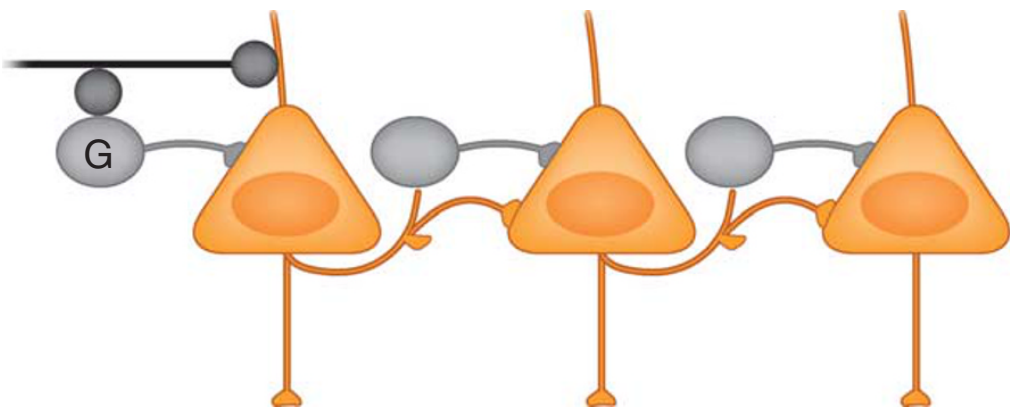

C
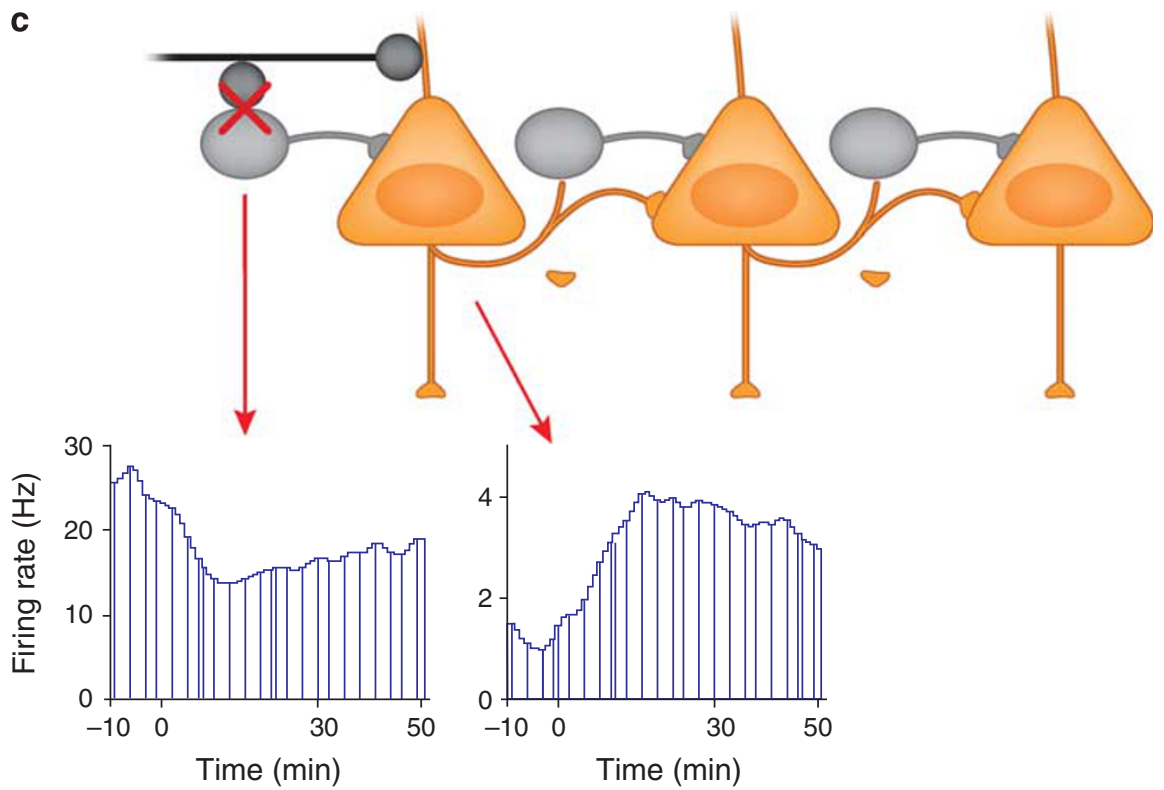

Figure 2. (a) Principal (P) or pyramidal cells in the neocortex and hippocampus, all which use glutamate as their neurotransmitter, receive extensive excitatory input from subcortical and cortical regions. In the absence of a counteracting inhibitory influence, activation of these inputs could cause a chain reaction of ever increasing excitation. (b) The regulation or stabilization of the firing of pyramidal cells is served by GABA (G) interneurons. One classic example of GABAergic influence is the feed-forward inhibition model where the effect of afferent excitation on a pyramidal neuron is dampened by co-activation of GABA interneurons that synapse onto the same pyramidal neuron. (c) The excitatory-inhibitory balance can be disrupted by many factors. An example is exposure to pro-psychotic compounds such as NMDA receptor antagonists. Blockade of NMDA receptors preferentially acts on fast spiking GABA interneurons because these neurons have a more depolarized membrane potential (note the higher firing rate on the recording from a putative GABA interneuron in the prefrontal cortex of an awake rat) and thus contain more open NMDA channels. This preferential inhibition of GABA interneurons creates an artificial state of disinhibition for the pyramidal cells and increases their firing rate.

NMDAR antagonist, there is a preferential effect on inhibiting the excitatory drive of GABA neurons compared with pyramidal neurons. This will produce an artificial state of disinhibition whereby the NMDAR antagonist produces a transient decrease in firing rate of GABA neurons that in turn enhances the firing of pyramidal neurons (Homayoun and Moghaddam, 2007b). The reduced impact of GABA on pyramidal neurons then would lead to an unstable situation where there is an artificial engagement of pyramidal neurons or noise as described by awake animal recordings (Jackson et al, 2004). This also may cause a secondary effect of enhanced glutamate release from local pyramidal cells, which then leads to excess activation of AMPA receptors at some synapse (Moghaddam et al, 1997). Overall, this process 
of NMDAR hypofunction-induced disinhibition may render the pyramidal neurons compromised when it comes to responding to incoming stimuli and passing on properly coordinated activity to subcortical regions.

\section{Postsynaptic Hypotheses}

On the postsynaptic level, the last 20 years of research has also led to an evolution in thinking about both causes and targets of NMDAR dysfunction. Functional NMDAR are heteroligomers composed of variable combinations of NR1, NRA-D, and NR3A-B subunits. Complex alterations in NMDAR subunit composition have been reported at both the protein and message level in schizophrenia, along with alterations in specific NMDAR-related postsynaptic proteins (Kristiansen et al, 2007). In addition, positive genetic associations are reported between both NR1 (Begni et al, 2003) and NR2B polymorphisms and schizophrenia (Martucci et al, 2006; Qin et al, 2005; Allen et al, 2008), suggesting that abnormalities of NMDAR expression or function may directly contribute to schizophrenia. NMDARs are primarily localized to postsynaptic dendritic terminals. However, additional populations exist on presynaptic terminals where they control glutamate release (Javitt et al, 1987; Corlew et al, 2008; Larsen et al, 2011) and growth cones of oligodendrocytes (Matute et al, 2005), and where dysfunction may be responsible for the well-replicated white matter abnormalities in schizophrenia (Ardekani et al, 2005). Nevertheless, the role of specific NMDAR subunits remains to be determined.

Along with intrinsic NMDAR dysfunction, disturbances in several modulatory mechanisms also have been demonstrated in schizophrenia, and have increasingly become targets of both etiological and therapeutic interventions (Figure 3). These include disturbances in synthesis and degradation of glycine and D-serine, which bind to the glycine modulatory site of the NMDAR (Javitt, 2007), and glutathione, which regulates the redox site (Gysin et al, 2007). In addition, endogenous inhibitors, such as kynurenic acid, may have a key role and thus may represent secondary targets for drug development (Wonodi and Schwarcz, 2010).

Glycine metabolism. Glycine in brain is synthesized primarily from L-serine by serine hydroxymethyltransferase, and regulated synaptically by glycine (GlyT1) transporters (Javitt, 2007). To date, there is limited evidence for disturbance of glycine metabolism in schizophrenia. Nevertheless, GlyT1 transporters may be an appropriate target for therapeutic intervention, potentially raising synaptic glycine to super-physiological levels in order to compensate for disturbances elsewhere in the system (Javitt, 2009a).

$D$-serine metabolism. In contrast, abnormalities in D-serine metabolism have been demonstrated at both the synthetic and degratory level. D-serine is synthesized in brain from $\mathrm{L}$-serine by serine racemase, and degraded by $\mathrm{D}$-amino-acid oxidase, which, in turn, is modulated by the protein G72. Genetic studies have shown associations of both enzymes with schizophrenia. Furthermore, serine racemase knockout mice show behavioral and structural abnormalities similar to those observed in schizophrenia, with a phenotype that can be rescued by crossbreeding with D-amino-acid oxidase (DAAO) knockouts. Perhaps even more important, reductions in D-serine levels have been demonstrated in both plasma and CSF in schizophrenia, suggesting potential physiological relevance to the genetic abnormalities (Labrie and Roder, 2009).

Glutathione/n-acetylcysteine. Genetic studies have implicated impairments in the glutathione system (Gysin et al, 2007). As with D-serine, reduced glutathione levels have been demonstrated in schizophrenia using both MRS and CSF measurements. Recently, associations also have been reported for several of the glutathione synthetic enzymes (Rodriguez-Santiago et al, 2010). Many factors nonspecifically affect brain glutathione levels. Thus, the glutathione site may represent a point of convergence for nonspecific brain injury mechanisms. Brain glutathione levels may be modulated to some extent by administration of dietary precursors, such as N-acetylcysteine (Dodd et al, 2008; Berk et al, 2008). To date, however, no high-affinity compounds have been developed that may function via modulation of this site.

\section{ALLOSTERIC MODULATORY SITES}

A critical concern with the overall approach of manipulating glutamate neurotransmission at presynaptic or postsynaptic levels is that traditional agonist or antagonist therapy may be detrimental. This is because glutamate synapses are highly dynamic synapses where stimulusinduced release of glutamate causes a rapid postsynaptic response and efficient uptake of either amino acid from the synaptic cleft. A sustained activation of receptors that mediate glutamate neurotransmission could result in neurotoxicity or adaptive responses that may be detrimental to cortical function. Thus, a more practical approach is to modulate the function of these receptors in an activitydependent manner, ie, to enhance or reduce their function transiently in response to an incoming stimulus. This function is served naturally in the brain by the so-called allosteric modulatory sites on many brain receptors. These sites, when activated, enhance the function of the natural neurotransmitters in stimulating the targeted receptor. In other words, they only work to modulate the function of the receptor when the receptor is stimulated by the natural neurotransmitter.

These targets can be ideal for treatment because, as depicted in Figure 4, unlike a direct agonist that sustains a continuous level of receptor activation, stimulation of these sites by so-called positive allosteric modulator (PAM) only potentiates the function of the natural ligand and thus only 


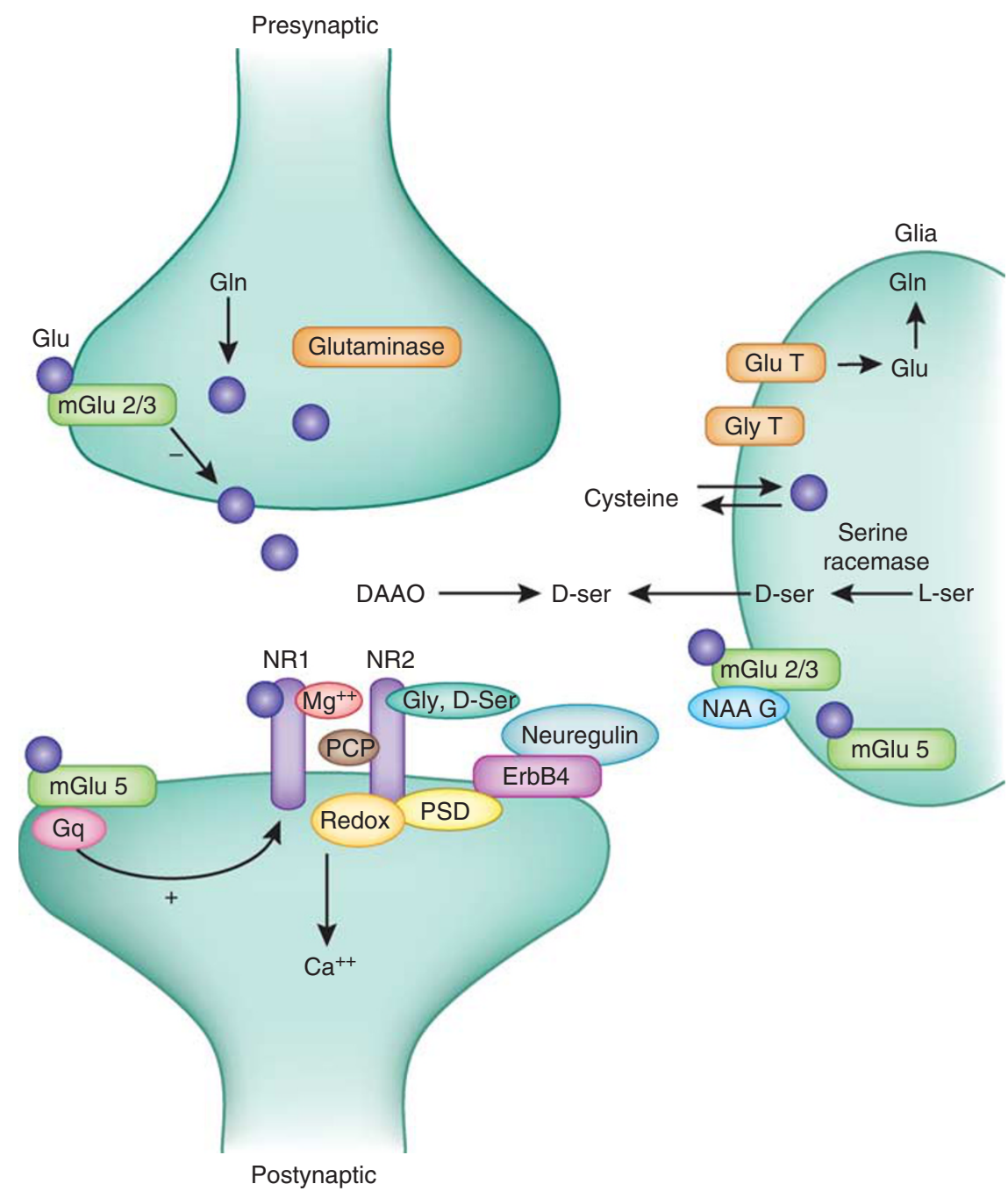

Figure 3. A simplified model of glutamate (Glu) synapse depicting some of the potential targets for manipulating the function of NMDA receptors. The two primary subunits of the receptor (NR1 and NR2) are depicted. On the presynaptic side, excess release of glutamate can be reduced by metabotropic group 2 receptors. Levels of vesicular glutamate also can be manipulated by the activity of the synthetic enzyme glutaminase, which converts glutamine (GIn) to Glu. On the postsynaptic site, several regulatory sites on the NMDA channel itself (eg, magnesium and PCP-binding sites, the D-serine and glycine (Gly) site and the redox (glutathione)) regulate the function of the receptor. In addition, other membrane-spanning receptors, such as the metabotropic group 5 (mGlu5) receptor or the ErbB4 receptor, indirectly influence the function of NMDAR by interacting through postsynaptic density (PSD) or signal transduction mechanisms. The glia includes a large number of proteins that influence both presynaptic and postsynaptic function of this synapse. These include transporters for both Glu and Gly, the D-serine-synthesizing enzyme serine racemase, D-serine transporter, as well as cystine-Glu transporter. In addition, a number of metabotropic Glu receptors including mGluR3 and mGluR5 are expressed by glia.

'stimulates' when it is activated by the natural neurotransmitter released in response to stimuli. In general, two sets of allosteric modulatory sites can be considered. First, those inherent to the NMDAR itself and second, those inherent to receptors that may serve to modulate NMDAR-mediated neurotransmission.

\section{Intrinsic Sites}

To date, the sites that have been most investigated in therapeutic trials are the glycine modulatory site and, to a much lesser extent, the redox site. Studies of glycine-site agonists have been possible because the two endogenous ligands for this site, glycine and D-serine, are both natural substances and so can be used in clinical trials even in advance of availability of optimized ligands. D-cycloserine, an antituberculosis drug, fortuitously cross-reacts with NMDAR, but is only a partial agonist. Overall, significant effects have been observed in several single-site studies of both glycine and D-serine (Javitt et al, 1994; Heresco-Levy et al, 1999; Tuominen et al, 2005; Tsai and Lin, 2010). To date, however, multicenter studies have not shown separation vs placebo (Buchanan et al, 2007; Weiser et al, 2008). However, both studies also showed substantial placebo effects. In the absence of an active comparator, therefore, it cannot be determined whether these should be interpreted as negative or failed studies. Caution, moreover, must be exercised in interpreting these studies, however, as none have been conducted with the types of internal controls typical of industry-sponsored studies. Most particularly, 


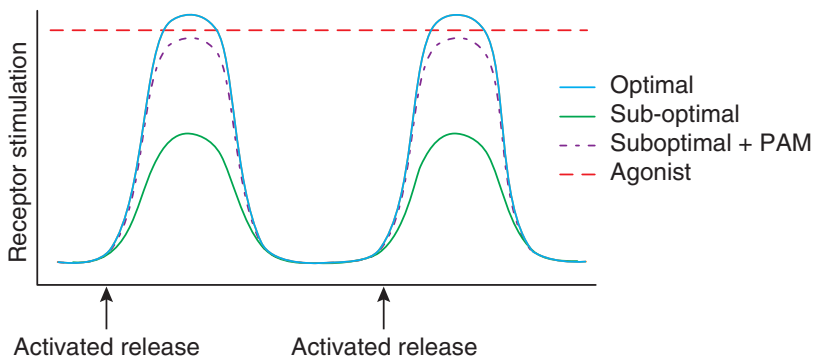

Figure 4. Allosteric modulation of receptors can selectively modulate active synapses. Under normal conditions, postsynaptic receptors are activated phasically when an action potential releases neurotransmitter from presynaptic terminals. A suboptimal activation of these receptors in a disease state is better treated with an allosteric positive modulator (PAM) that enhances the function of the natural neurotransmitter on those receptors as opposed to an agonist that produces constant activation of receptors. The latter could lead to desensitization of receptors, neurotoxicity, and other side effects.

compounds have been used at non-optimized doses. In the case of glycine, the maximum dose use has been limited by general tolerability; in the case of D-serine, by nephrotoxicity. As no target engagement biomarkers have been used in these studies, the degree of target occupancy remains unknown.

Glycine transport inhibitors: basic mechanisms. An alternative approach to glycine modulation was first proposed in the late 1990's based upon analogy to the serotonin system in depression. The goal of this approach was to increase synaptic glycine levels by blocking function of the GlyT1 that are co-localized with NDMAR, thereby permitting a natural increase in synaptic glycine levels (Javitt, 2004). Initial preclinical studies were performed with glycyldodecylamide and related compounds, which were shown to reverse PCP-induced hyperactivity with parallel rank order of potency to their effects on glycine transport (Javitt and Frusciante, 1997; Javitt et al, 1999). Subsequently, higher affinity compounds such as N[3-(4'fluorophenyl)-3-(4'-phenylphenoxy)propyl]sarcosine (NFPS) were found to modulate hippocampal NMDAR function in vitro (Bergeron et al, 1998), and to modulate striatal dopamine release (Javitt et al, 2004) and prefrontal cortical activity (Chen et al, 2003) in vivo.

Since then, several series of high-affinity glycine transport inhibitors have been developed and shown to be effective in multiple animal models related to schizophrenia (Javitt, 2009 a, b). Although initial high-affinity compounds, such as NFPS, showed unexpected toxicity such as compulsive walking ('obstinate progression') and respiratory distress in initial in vivo animal studies, these side effects were subsequently shown not to be NMDAR mediated (Kopec et al, 2010). In general, recently developed non-sarcosinebased competitive GlyT1 antagonists show greater preclinical safety and tolerability than initial sarcosine-based compounds, such as NFPS, that showed irreversible, noncompetitive inhibition of GlyT1 function (Javitt, 2009a; Wolkenberg and Sur, 2010).
Glycine transport inhibitors: clinical studies. Initial clinical studies were performed with the naturally occurring glycine transport inhibitor sarcosine (N-methylglycine), which has been found to be effective in both acute and chronic schizophrenia in several small-scale studies conducted in Taiwan (Lane et al, 2008; Lane et al, 2005; Lane et al, 2010; Tsai et al, 2004). As with glycine and D-serine, no biomarkers were available to demonstrate engagement of the glycinebinding site; therefore, there is no way to know whether the dose used represents a clinically optimal dose.

Most recently, the first selective, high-affinity GlyT1 compound, RG-1678 (Roche), was studied in a phase II program involving 323 subjects (Umbricht et al, 2010). As opposed to earlier studies, the clinical dose of RG-1678 was selected based upon a PET study of glycine-site occupancy, with dose chosen to prevent activation-related NMDAR desensitization. The study demonstrated, first, that inhibition of GlyT1-mediated transport does indeed lead to increased CNS glycine levels, and second, that resultant allosteric NMDAR via the glycine modulatory site may be therapeutically beneficial. This compound recently has been entered into definitive phase III trials for treatment of persistent negative symptoms.

$D A A O$ inhibition. In the case of D-serine, a 'second generation' approach also is under development. In this approach, D-serine is combined with a DAAO inhibitor to prevent renal and brain D-serine degradation. Use of this approach produces a 30 -fold increase in D-serine potency in animal models (Hashimoto et al, 2009), potentially decreasing clinically effective doses of $\mathrm{D}$-serine from gram to milligram levels. As knock out of renal DAAO also prevents D-serine toxicity (Konno et al, 2010), it is possible that a combination treatment also will produce greater compound tolerability. To date, however, DAAO inhibitors remain in the preclinical testing stage, so ultimate utility of this approach remains to be determined. Finally, the cystine/ glutamate antiporter (xCT) may be crucial in the regulation of brain glutathione levels, and may serve as an additional target for glutamate-related drug development (Shih et al, 2006).

Glutamatergic basis of clozapine response. Finally, studies with NMDAR may shed light on the mechanism by which the atypical antipsychotic clozapine is differentiated from other typical and atypical antipsychotics. Clozapine effectively reduces the impact of NMDA receptor antagonists on cortical neuron hyperactivity (Homayoun and Moghaddam, 2007a). Among its many pharmacological effects, clozapine significantly potentiates NMDAR transmission in the brain, by inhibition of system A-type glycine transporters in the brain (Javitt et al, 2004). Similarly, clozapine, along with D-serine and GlyT1 inhibitors, block PCP effects on social recognition (Shimazaki et al, 2010) and other rodent models (Lipina et al, 2005). Finally, while glycine, D-serine, and sarcosine have found to be effective in combination with typical antipsychotics or newer atypicals such as risperidone 
or olanzapine, they appear less effective when combined with clozapine (Tsai and Lin, 2010). This lack of effect may reflect that clozapine already functions, at least in part, as a NMDAR/glycine-site agonist.

\section{Extrinsic Sites}

A second approach to enhance NMDAR is by targeting metabotropic glutamate receptors, which, in turn, may modulate either glutamate presynaptically or NMDAR postsynaptically. Presynaptic glutamate release is modulated by $\mathrm{mGlu} 2 / 3$ receptors, which serve to limit release. To date, clinical trials have been conducted with the mGlu2/3 full agonist, which has shown promising results. However, as with all full agonists, downregulation of receptors over time also is a concern. As opposed to full agonists, therefore, PAMs for this site may maximize efficacy while limiting side effects and toxicity.

At the postsynaptic level, mGlu 5 receptors may provide an indirect target for modulation of NMDAR. This group of receptors, which at some synapses are localized near NMDARs, modulate the dynamics of NMDAR channels by increasing NMDAR-mediated current (Conn et al, 2009). Targeting the mGlu5 receptors with PAMs has shown promise in some preclinical models (Lecourtier et al, 2007; Liu et al, 2008). A number of mGluR5 agonists and PAMs with appropriate pharmacological properties have been synthesized over recent years, and have been shown to be effective in specific preclinical models of schizophrenia, although issues related to regional expression and potential downregulation during chronic treatment need to be resolved (Parmentier-Batteur et al, 2010; RodriguezSantiago et al, 2010; Spear et al, 2010). In addition to direct targeting of the receptor, other novel targets such as Norbin, an endogenous protein that interacts with mGlu5 receptors in vivo, have been proposed (Wang et al, 2009).

In addition to mGlu receptors, other interesting targets related to cortical circuits have been proposed (Wroblewska and Lewis, 2009; Marek et al, 2010). These include agents that stimulate AMPA-type glutamate receptors or 'AMPAkines' (Arai and Kessler, 2007), although studies to date have not shown efficacy for either symptoms or cognition (Goff et al, 2008). Other compounds, such as the M1/M4 muscarinic agent xanomeline (Bridges et al, 2010) and neurosteroids such as pregnenolone sulfate (Marx et al, 2009; Ritsner, 2010) may also function in part through indirect modulation of NMDAR-mediated neurotransmission. Thus, continued research into mechanisms of normal and abnormal NMDAR regulation may lead to further advances in drug development in schizophrenia.

\section{FUTURE DIRECTIONS}

Although the dopamine model of schizophrenia remains heuristically valuable, many aspects of schizophrenia cannot be explained based upon dopaminergic dysfunction alone, and many patients with schizophrenia remain persistently disabled despite treatment with various dopaminergic compounds. Glutamatergic theories of schizophrenia account for negative symptoms and cognitive dysfunction, as well as positive symptoms, and thus may lead to new treatment approaches specifically targeting this unmet medical need. Improving the future treatment of schizophrenia and increasing our biological understanding of the disease will be contingent on development of appropriate models and biomarkers for glutamatergic drug development. In particular, studies focusing on mechanistically based and clinically relevant dynamic circuit models are needed to consolidate the evolving genetic data with translational physiological measures. Although clinical drug development progresses slowly, the field has now progressed to the point where treatment predictions of the glutamate model can be tested.

\section{ACKNOWLEDGEMENTS}

Funded in part by grants R37 (MH48404), R01 (MH084906), Pittsburgh Life Sciences Greenhouse (Bita Moghaddam), and R01DA03383 and P50 MH086385 (Daniel Javitt). This work was supported by National Institute of Mental Health and the Pittsburgh Life Sciences Greenhouse.

\section{DISCLOSURE}

Bita Moghaddam declares no conflict of interest. Daniel Javitt holds intellectual property rights for use of glycine, D-serine, and glycine transport inhibitors in treatment of schizophrenia and equity interest in Glytech. Within the past year, Dr Daniel Javitt has served as a consultant to Sepracor, AstraZeneca, Pfizer, Cypress Bioscience, Merck, Sunovion, Eli Lilly, and BMS, and has received research support from Pfizer, Roche, and Jazz Pharmaceuticals. Dr Daniel Javitt serves on the Scientific Advisory Board of Promentis.

\section{REFERENCES}

Abbott A (2010). Schizophrenia: the drug deadlock. Nature 468: 158-159.

Adams B, Moghaddam B (1998). Corticolimbic dopamine neurotransmission is temporally dissociated from the cognitive and locomotor effects of phencyclidine. J Neurosci 18: 5545-5554.

Allen NC, Bagade S, McQueen MB, loannidis JP, Kavvoura FK, Khoury MJ et al (2008). Systematic meta-analyses and field synopsis of genetic association studies in schizophrenia: the SzGene database. Nat Genet 40: 827-834.

Anderson S, Volk D et al (1996). Increased density of microtubule associated protein 2-immunoreactive neurons in the prefrontal white matter of schizophrenic subjects. Schizophr Res 19: 111-119.

Arai AC, Kessler M (2007). Pharmacology of ampakine modulators: from AMPA receptors to synapses and behavior. Curr Drug Targets 8: 583-602.

Ardekani BA, Bappal A et al (2005). Brain morphometry using diffusion-weighted magnetic resonance imaging: application to schizophrenia. Neuroreport 16: 1455-1459.

Battaglia G, Monn JA et al (1997). In vivo inhibition of veratridine-evoked release of striatal excitatory amino acids by the group II metabotropic glutamate receptor agonist LY354740 in rats. Neurosci Lett 229: 161-164. This brief report was critical in establishing that exogenous mGlu2/3 receptor activation normalizes excessive release of glutamate. 
Begni S, Moraschi S et al (2003). Association between the G1001C polymorphism in the GRIN1 gene promoter region and schizophrenia. Biol Psychiatry 53: 617-619.

Bergeron R, Meyer T et al (1998). Modulation of N-methyl-D-aspartate receptor function by glycine transport. Proc Natl Acad Sci USA 95: 15730-15734.

Berk M, Copolov D et al (2008). N-acetyl cysteine as a glutathione precursor for schizophrenia - a double-blind, randomized, placebo-controlled trial. Biol Psychiatry 64: 361-368.

Breier A, Malhotra AK et al (1997). Association of ketamine-induced psychosis with focal activation of the prefrontal cortex in healthy volunteers. Am J Psychiatry 154: 805-811.

Bridges TM, LeBois EP et al (2010). The antipsychotic potential of muscarinic allosteric modulation. Drug News Perspect 23: 229-240.

Buchanan RW, Javitt DC et al (2007). The Cognitive and Negative Symptoms in Schizophrenia Trial (CONSIST): the efficacy of glutamatergic agents for negative symptoms and cognitive impairments. Am J Psychiatry 164: 1593-1602.

Buzsaki G, Geisler C et al (2004). Interneuron diversity series: circuit complexity and axon wiring economy of cortical interneurons. Trends Neurosci 27: 186-193.

Carlsson M, Carlsson A (1989). The NMDA antagonist MK-801 causes marked locomotor stimulation in monamine-depleted mice. J Neural Transm 75: 221-226. This is one of the first studies demonstrating that adverse behavioral effects of NMDA receptor antagonists persist in the absence of dopamine.

Cartmell J, Monn JA et al (1999). The metabotropic glutamate 2/3 receptor agonists LY354740 and LY379268 selectively attenuate phencyclidine versus d-amphetamine motor behaviors in rats. J Pharmacol Exp Ther 291: 161-170.

Cartmell J, Monn JA et al (2000). The mGlu(2/3) receptor agonist LY379268 selectively blocks amphetamine ambulations and rearing. Eur J Pharmacol 400: 221-224.

Chen GM, Weston JK (1960). The analgesic and anesthetic effect of 1-(1-phenylcyclohexyl) piperidine $\mathrm{HCl}$ on the monkey. Anesth Analg 39: 132-137.

Chen L, Muhlhauser M et al (2003). Glycine tranporter-1 blockade potentiates NMDA-mediated responses in rat prefrontal cortical neurons in vitro and in vivo. J Neurophysiol 89: 691-703.

Conn PJ, Lindsley CW et al (2009). Activation of metabotropic glutamate receptors as a novel approach for the treatment of schizophrenia. Trends Pharmacol Sci 30: 25-31. This is an excellent review on the potential of mGlu receptors for treatment of schizophrenia.

Corlew R, Brasier DJ et al (2008). Presynaptic NMDA receptors: newly appreciated roles in cortical synaptic function and plasticity. Neuroscientist 14: 609-625.

Creese I, Burt D et al (1976). Dopamine receptor binding predicts clinical and pharmacological potencies of antischizophrenic drugs. Science 192: 481-483.

Csicsvari J, Hirase $\mathrm{H}$ et al (1998). Reliability and state dependence of pyramidal cell-interneuron synapses in the hippocampus: an ensemble approach in the behaving rat. Neuron 21: 179-189.

Dodd S, Dean O et al (2008). N-acetylcysteine for antioxidant therapy: pharmacology and clinical utility. Expert Opin Biol Ther 8: 1955-1962.

Ehrlichman RS, Maxwell CR et al (2008). Deviance-elicited changes in eventrelated potentials are attenuated by ketamine in mice. J Cogn Neurosci 20: 1403-1414.

Foussias G, Remington G (2010). Antipsychotics and schizophrenia: from efficacy and effectiveness to clinical decision-making. Can J Psychiatry 55: 117-125.

Gaisler-Salomon I, Miller GM et al (2009). Glutaminase-deficient mice display hippocampal hypoactivity, insensitivity to pro-psychotic drugs and potentiated latent inhibition: relevance to schizophrenia. Neuropsychopharmacol 34: 2305-2322.

Geyer MA, Krebs-Thomson K et al (1999b). The effects of M100907 on pharmacological and developmental animal models of prepulse inhibition deficits in schizophrenia. Neuropsychopharmacology 21: S134-S142.

Goff DC, Lamberti JS et al (2008). A placebo-controlled add-on trial of the Ampakine, CX516, for cognitive deficits in schizophrenia. Neuropsychopharmacol 33: 465-472

Goldberg T, Weinberger D (1996). Effects of neuroleptic medications on the cognition of patients with schizophrenia: a review of recent studies. J Clin Psychiatry 57(Suppl 9): 62-65.

Goldberg TE, David A et al (2003). Neurocognitive deficits in schizophrenia. Schizophrenia. S. R. Hirsch and D. R. Weinberger. Blackwell Publishing Company: Oxford, 168-184

Green MF, Nuechterlein KH (1999). Should schizophrenia be treated as a neurocognitive disorder? Schizophr Bull 25: 309-319.

Gysin R, Kraftsik R et al (2007). Impaired glutathione synthesis in schizophrenia: convergent genetic and functional evidence. Proc Natl Acad Sci USA 104: $16621-16626$.
Hashimoto K, Fujita $\mathrm{Y}$ et al (2009). Co-administration of a D-amino acid oxidase inhibitor potentiates the efficacy of D-serine in attenuating prepulse inhibition deficits after administration of dizocilpine. Biol Psychiatry 65: 1103-1106.

Heekeren K, Daumann J et al (2008). Mismatch negativity generation in the human 5HT2A agonist and NMDA antagonist model of psychosis. Psychopharmacology 199: 77-88.

Heresco-Levy U, Javitt DC et al (1999). Efficacy of high-dose glycine in the treatment of enduring negative symptoms of schizophrenia. Arch Gen Psychiatry 56: 29-36.

Homayoun $\mathrm{H}$, Jackson ME et al (2005). Activation of metabotropic glutamate 2/3 receptors reverses the effects of NMDA receptor hypofunction on prefrontal cortex unit activity in awake rats. J Neurophysiol 93: 1989-2001.

Homayoun H, Moghaddam B (2007a). Fine-tuning of awake prefrontal cortex neurons by clozapine: comparison with haloperidol and $\mathrm{N}$-desmethylclozapine. Biol Psychiatry 61: 679-687.

Homayoun H, Moghaddam B (2007b). NMDA receptor hypofunction produces opposite effects on prefrontal cortex interneurons and pyramidal neurons. J Neurosci 27: 11496-11500.

Jackson M, Homayoun $\mathrm{H}$ et al (2004). NMDA receptor hypofunction produces concomitant firing rate potentiation and burst activity reduction in the prefrontal cortex. Proc Natl Acad Sci USA 101: 6391-6396. This was the first recording of the effect of NMDA receptor inhibition on awake cortical neurons demonstrating that, in contrast to brain slices or anesthetized animals, this inhibition increases spontaneous activity of cortical neurons.

Javitt DC (2004). Glutamate as a therapeutic target in psychiatric disorders. Mol Psychiatry 9: 984-997, 979.

Javitt DC (2007). Glutamate and schizophrenia: phencyclidine, N-methyl-Daspartate receptors, and dopamine-glutamate interactions. Int Rev Neurobiol 78: 69-108.

Javitt DC (2009a). Glycine transport inhibitors for the treatment of schizophrenia: symptom and disease modification. Curr Opin Drug Discov Devel 12: 468-478. Recent review of the glycine transport inhibitor approach.

Javitt DC (2009b). When doors of perception close: bottom-up models of disrupted cognition in schizophrenia. Annu Rev Clin Psychol 5: 249-275.

Javitt DC, Balla A et al (2004). Reversal of phencyclidine-induced dopaminergic dysregulation by N-methyl-D-aspartate receptor/glycine-site agonists. Neuropsychopharmacology 29: 300-307. Demonstration that schizophrenia-like dopaminergic abnormalities can be induced by NMDAR antagonists and that the effect can be blocked by NMDAR/glycine site agonists.

Javitt DC, Balla A et al (1999). A.E. Bennett Research Award. Reversal of phencyclidine-induced effects by glycine and glycine transport inhibitors. Biol Psychiatry 45: 668-679.

Javitt DC, Doneshka P et al (1993). Impairment of early cortical processing in schizophrenia: an event-related potential confirmation study. Biol Psychiatry 33: 513-519.

Javitt DC, Frusciante M (1997). Glycyldodecylamide, a phencyclidine behavioral antagonist, blocks cortical glycine uptake: implications for schizophrenia and substance abuse. Psychopharmacology 129: 96-98.

Javitt DC, Jotkowitz A et al (1987). Non-competitive regulation of phencyclidine/ sigma-receptors by the $\mathrm{N}$-methyl-D-aspartate receptor antagonist D-(-)-2amino-5-phosphonovaleric acid. Neurosci Lett 78: 193-198.

Javitt DC, Steinschneider M et al (1996). Role of cortical N-methyl-D-aspartate receptors in auditory sensory memory and mismatch negativity generation: implications for schizophrenia. Proc Natl Acad Sci USA 93: 11962-11967.

Javitt DC, Zukin SR (1991). Recent advances in the phencyclidine model of schizophrenia. Am J Psychiatry 148: 1301-1308.

Javitt DC, Zylberman I et al (1994). Amelioration of negative symptoms in schizophrenia by glycine. Am J Psychiatry 151: 1234-1236. First report of effectiveness of a glycine-site agonist in schizophrenia.

Kim J, Kornhuber $\mathrm{H}$ et al (1980). Low cerebrospinal fluid glutamate in schizophrenic patients and a new hypothesis on schizophrenia. Neurosci Lett 20: 379-382

Kinon BJ, Zhang $L$ et al (2011). A multicenter, inpatient, phase 2, double-blind, placebo-controlled dose-ranging study of LY2140023 monohydrate in patients with DSM-IV schizophrenia. J Clin Psychopharmacol 31: 349-355.

Kirkpatrick B, Fenton WS et al (2006). The NIMH-MATRICS consensus statement on negative symptoms. Schizophr Bull 32: 214-219.

Konno R, Hamase K et al (2010). Mutant mice and rats lacking D-amino acid oxidase. Chem Biodiversity 7: 1450-1458.

Kopec K, Flood DG et al (2010). Glycine transporter (GlyT1) inhibitors with reduced residence time increase prepulse inhibition without inducing hyperlocomotion in DBA/2 mice. Biochem Pharmacol 80: 1407-1417.

Kristiansen LV, Huerta l et al (2007). NMDA receptors and schizophrenia. Curr Opin Pharmacol 7: 48-55. 
Krystal J, Karper L et al (1995). Modulating ketamine-induced thought disorder with lorazepam and haloperidol in humans. Schizophr Res 15: 156a.

Krystal JH, Abi-Saab W et al (2005). Preliminary evidence of attenuation of the disruptive effects of the NMDA glutamate receptor antagonist, ketamine, on working memory by pretreatment with the group II metabotropic glutamate receptor agonist, LY354740, in healthy human subjects. Psychopharmacology (Berl) 179: 303-309. First human data supporting use of mGluR2/3 agonists in a clinical model of schizophrenia.

Krystal JH, Karper LP et al (1994). Subanesthetic effects of the noncompetitive NMDA antagonist, ketamine, in humans. Psychotomimetic, perceptual, cognitive, and neuroendocrine responses. Arch Gen Psychiatry 51: 199-214.

Labrie V, Roder JC (2009). The involvement of the NMDA receptor D-serine/glycine site in the pathophysiology and treatment of schizophrenia. Neurosci Biobehav Rev 34: 351-372. Recent comprehensive review of D-serine metabolic disturbances in schizophrenia.

Lane HY, Chang YC et al (2005). Sarcosine or D-serine add-on treatment for acute exacerbation of schizophrenia: a randomized, double-blind, placebo-controlled study. Arch Gen Psychiatry 62: 1196-1204.

Lane HY, Lin CH et al (2010). A randomized, double-blind, placebo-controlled comparison study of sarcosine ( $\mathrm{N}$-methylglycine) and D-serine add-on treatment for schizophrenia. Int J Neuropsychopharmacol 13: 451-460.

Lane HY, Liu YC et al (2008). Sarcosine ( $\mathrm{N}$-methylglycine) treatment for acute schizophrenia: a randomized, double-blind study. Biol Psychiatry 63: 9-12.

Larsen RS, Corlew RJ, Henson MA, Roberts AC, Mishina M, Watanabe M et al (2011). NR3A-containing NMDARs promote neurotransmitter release and spike timing-dependent plasticity. Nat Neurosci 14: 338-344.

Lecourtier L, Homayoun $\mathrm{H}$ et al (2007). Positive allosteric modulation of metabotropic glutamate 5 (mGlu5) receptors reverses N-Methyl-D-aspartate antagonist-induced alteration of neuronal firing in prefrontal cortex. Biol Psychiatry 62: 739-746.

Lesh TA, Niendam TA et al (2011). Cognitive control deficits in schizophrenia: mechanisms and meaning. Neuropsychopharmacol 36: 316-338.

Lieberman JA, Stroup TS et al (2005). Effectiveness of antipsychotic drugs in patients with chronic schizophrenia. N Engl J Med 353: 1209-1223.

Lipina T, Labrie V, Weiner I, Roder J (2005). Modulators of the glycine site on NMDA receptors, D-serine and ALX 5407, display similar beneficial effects to clozapine in mouse models of schizophrenia. Psychopharmacology (Berl) 179: 54-67.

Liu F, Grauer S et al (2008). ADX47273 [S-(4-fluoro-phenyl)-\{3-[3-(4-fluoro-phenyl)[1,2,4]-oxadiazol-5-yl]-piper idin-1-yl\}-methanone]: a novel metabotropic glutamate receptor 5 -selective positive allosteric modulator with preclinical antipsychotic-like and procognitive activities. J Pharmacol Exp Ther 327: 827-839.

Liu J, Moghaddam B (1995). Regulation of glutamate efflux by excitatory amino acid receptors: evidence for tonic inhibitory and phasic excitatory regulation. J Pharmacol Exp Ther 274: 1209-1215.

Lorrain DS, Baccei CS et al (2003). Effects of ketamine and N-methyl-D-aspartate on glutamate and dopamine release in the rat prefrontal cortex: modulation by a group III selective metabotropic glutamate receptor agonist LY379268. Neuroscience 117: 697-706.

Luby ED, Gottlieb JS et al (1962). Model psychoses and schizophrenia. Am J Psychiatry 119: 61-67.

Maccaferri G, Dingledine R (2002). Control of feedforward dendritic inhibition by NMDA receptor-dependent spike timing in hippocampal interneurons. J Neurosci 22: 5462-5472.

Marder SR (1999). Limitations of dopamine-D2 antagonists and the search for novel antipsychotic strategies. Neuropharmacology 21: S117-S121.

Marek GJ, Behl B et al (2010). Glutamatergic (N-methyl-D-aspartate receptor) hypofrontality in schizophrenia: too little juice or a miswired brain? Mol Pharmacol 77: 317-326.

Martucci L, Wong AH et al (2006). N-methyl-D-aspartate receptor NR2B subunit gene GRIN2B in schizophrenia and bipolar disorder: polymorphisms and mRNA levels. Schizophr Res 84: 214-221.

Marx CE, Keefe RS et al (2009). Proof-of-concept trial with the neurosteroid pregnenolone targeting cognitive and negative symptoms in schizophrenia. Neuropsychopharmacol 34: 1885-1903.

Matute C, Melone $\mathrm{M}$ et al (2005). Increased expression of the astrocytic glutamate transporter GLT-1 in the prefrontal cortex of schizophrenics. Glia 49: 451-455.

Meltzer HY, Arvanitis L et al (2004). Placebo-controlled evaluation of four novel compounds for the treatment of schizophrenia and schizoaffective disorder. Am J Psychiatry 161: 975-984.

Moghaddam B (2003). Bringing order to the glutamate chaos in schizophrenia. Neuron 40: 881-884.

Moghaddam B (2004). Targeting metabotropic glutamate receptors for treatment of the cognitive symptoms of schizophrenia. Psychopharmacology (Berl) 174: $39-44$.
Moghaddam B, Adams B (1998). Reversal of phencyclidine effects by a group II metabotropic glutamate receptor agonist in rats. Science 281: 1349-1352.

Moghaddam B, Adams B et al (1997). Activation of glutamatergic neurotransmission by ketamine: a novel step in the pathway from NMDA receptor blockade to dopaminergic and cognitive disruptions associated with the prefrontal cortex. J Neurosci 17: 2921-2927.

Muir J, Everitt B et al (1995). Reversal of visual attentional dysfunction following lesions of the cholinergic basal forebrain by physostigmine and nicotine but not by the 5-HT3 receptor antagonist, ondansetron. Psychopharmacology 118: 82-92.

Naatanen R, Kahkonen S (2009). Central auditory dysfunction in schizophrenia as revealed by the mismatch negativity (MMN) and its magnetic equivalent $\mathrm{MMNm}$ : a review. Int J Neuropsychopharmacol/ 12: 125-135.

Omdal R, Brokstad $\mathrm{K}$ et al (2005). Neuropsychiatric disturbances in SLE are associated with antibodies against NMDA receptors. Eur $J$ Neurol 12: 392-398.

Parmentier-Batteur S, OBrien JA, Doran S, Nguyen SJ, Flick RB, Uslaner JM (2010). Differential effects of the mGluR5 positive allosteric modulator CDPPB in the cortex and striatum following repeated administration. Neuropharmacology (e-pub ahead of print 26 November 2010).

Patil ST, Zhang $L$ et al (2007). Activation of mGlu2/3 receptors as a new approach to treat schizophrenia: a randomized Phase 2 clinical trial. Nat Med 13 1102-1107.

Preskorn SH, Baker B et al (2008). An innovative design to establish proof of concept of the antidepressant effects of the NR2B subunit selective N-methyl-Daspartate antagonist, CP-101,606, in patients with treatment-refractory major depressive disorder. J Clin Psychopharmacol 28: 631-637.

Qin S, Zhao X et al (2005). An association study of the N-methyl-D-aspartate receptor NR1 subunit gene (GRIN1) and NR2B subunit gene (GRIN2B) in schizophrenia with universal DNA microarray. Eur J Hum Genet 13: 807-814.

Ritsner MS (2010). Pregnenolone, dehydroepiandrosterone, and schizophrenia: alterations and clinical trials. CNS Neurosci Ther 16: 32-44.

Rodriguez-Santiago B, Brunet A et al (2010). Association of common copy number variants at the glutathione S-transferase genes and rare novel genomic changes with schizophrenia. Mol Psychiatry 15: 1023-1033.

Schoepp DD, Johnson BG et al (1997). LY354740 is a potent and highly selective group II metabotropic glutamate receptor agonist in cells expressing human glutamate receptors. Neuropharmacology 36: 1-11.

Seeman P (2010). Dopamine D2 receptors as treatment targets in schizophrenia. Clini Schizophr Relat Psychoses 4: 56-73.

Seeman P, Chau-Wong M et al (1975). Brain receptors for antipsychotic drugs and dopamine: direct binding assays. Proc Natl Acad Sci USA 72: 4376-4380.

Shimazaki T, Kaku A, Chaki S (2010). D-Serine and a glycine transporter-1 inhibitor enhance social memory in rats. Psychopharmacology 209: 263-270.

Shih AY, Erb H et al (2006). Cystine/glutamate exchange modulates glutathione supply for neuroprotection from oxidative stress and cell proliferation. J Neurosci 26: $10514-10523$.

Simpson EH, Kellendonk C et al (2010). A possible role for the striatum in the pathogenesis of the cognitive symptoms of schizophrenia. Neuron 65: 585-596.

Spear N, Gadient RA, Wilkins DE, Do M, Smith JS, Zeller KL (2010). Preclinical profile of a novel metabotropic glutamate receptor 5 positive allosteric modulator. Eur J Pharmacol 659: 146-154.

Tikhonravov D, Neuvonen T et al (2008). Effects of an NMDA-receptor antagonist MK-801 on an MMN-like response recorded in anesthetized rats. Brain Res 1203: 97-102.

Tsai G, Lane HY et al (2004). Glycine transporter I inhibitor, N-methylglycine (sarcosine), added to antipsychotics for the treatment of schizophrenia. Biol Psychiatry 55: 452-456. First report of efficacy of the naturally occurring glycine transport inhibitor sarcosin in schizophrenia.

Tsai GE, Lin PY (2010). Strategies to enhance N-methyl-D-aspartate receptormediated neurotransmission in schizophrenia, a critical review and metaanalysis. Curr Pharm Des 16: 522-537.

Tuominen $\mathrm{H}$, Tiihonen $\mathrm{J}$ et al (2005). Glutamatergic drugs for schizophrenia: a systematic review and meta-analysis. Schizophr Res 72: 225-234.

Umbricht D, Schmid L et al (2000). Ketamine-induced deficits in auditory and visual context-dependent processing in healthy volunteers: implications for models of cognitive deficits in schizophrenia. Arch Gen Psychiatry 57: 1139-1147.

Umbricht D, Yoo K et al (2010). Glycine transporter type 1 (GLYT1) inhibitor RG1678: positive results of the proof-of-concept study for the treatment of negative symptoms in schizophrenia. Neuropsychopharmacology 35: S320-S321.

Vollenweider FX, Leenders KL et al (1997). Differential psychopathology and patterns of cerebral glucose utilisation produced by (S)- and (R)-ketamine in 
healthy volunteers using positron emission tomography (PET). Eur Neuropsychopharmacol 7: 25-38.

Wang $\mathrm{H}$, Westin L et al (2009). Norbin is an endogenous regulator of metabotropic glutamate receptor 5 signaling. Science 326: 1554-1557.

Weiser et al (2008). Subtle cognitive dysfunction in nonaffected siblings of individuals affected by nonpsychotic disorders. Biol Psych 63: 602-608.

Wolkenberg SE, Sur C (2010). Recent progress in the discovery of non-sarcosine based GlyT1 inhibitors. Curr Top Med Chem 10: 170-186.
Wonodi I, Schwarcz R (2010). Cortical kynurenine pathway metabolism: a novel target for cognitive enhancement in schizophrenia. Schizophr Bull 36: 211-218. Authoratative review concerning potential involvement of kynurenic acid abnormalities in schizophrenia.

Wroblewska B, Lewis DA (2009). Validating novel targets for pharmacological interventions in schizophrenia. Am J Psychiatry 166: 753-756.

Zukin SR, Zukin RS (1979). Specific [3H]phencyclidine binding in rat central nervous system. Proc Natl Acad Sci USA 76: 5372-5376. 